\section{Christoph Bachmann}

\section{Die Cochrane Collaboration}

Die Cochrane Collaboration ist eine internationale gemeinnützige Organisation, die wissenschaftliche Forschung auf der Basis der evidenzbasierten Medizin betreibt. Sie wurde 1993 gegründet und ist nach dem britischen Epidemiologen Sir Archibald Leman Cochrane (19091988) benannt. Die Organisation strebt eine Optimierung der wissenschaftlichen Erkenntnisse als Entscheidungsgrundlage für behandelnde Ärzte an. Dazu führt die Cochrane Collaboration Meta-Analysen, d.h. systematische Übersichtsarbeiten («systematic reviews»), auf der Basis von kontrollierten klinischen Studien durch. Diese Reviews werden in einer vierteljährlich aktualisierten Datenbank namens Cochrane Database of Systematic Reviews publiziert und tragen das bekannte Logo der Cochrane Collaboration, dessen innerer Teil das Auswertungsdiagramm einer Meta-Analyse darstellt.

\section{Cochrane Reviews und Phytotherapie}

Seit der Jahrtausendwende sind unter dem Stichwort «phytotherapy» etwa 100 Reviews der Cochrane Collaboration publiziert worden. Die Organisation unterscheidet aber nicht zwischen westlicher Phytotherapie und Traditioneller Chinesischer Medizin (TCM). Cochrane Reviews, die sich mit TCM befassen, machen etwa ein Drittel der aufgeführten Publikationen aus. Einige weitere Reviews befassen sich entweder mit Homöopathie, was gar nicht unter das Stichwort «phytotherapy» passt, mit Ayur-

\title{
Cochrane Reviews und pflanzliche Arzneimittel: Zum Teil positive Resultate
}

Reviews der Cochrane Collaboration geniessen in der medizinischen Fachwelt höchstes Ansehen, und ihre Schlussfolgerungen gelten als fast endgültiges Urteil über die Wirksamkeit oder eben Unwirksamkeit einer Therapie. Die Autoren von Cochrane Reviews über phytotherapeutische Behandlungen spiegeln die Haltung der Mehrheit der Vertreter der traditionellen Medizin wider: grosse Skepsis. In der Folge werden die Resultate einiger Reviews vorgestellt.

vedischer Medizin oder mit anderen Anwendungen, die nicht wirklich zur Phytotherapie gehören. So kann man 50-60 Reviews als eigentlich phytotherapeutisch bezeichnen. In der Folge werden übersichtsmässig einige dieser Publikationen vorgestellt, die in den letzten Jahren publiziert wurden und die Wirksamkeit einiger sehr wichtiger Arzneipflanzen der westlichen Phytotherapie beurteilen. Diese spiegeln die allgemeine, sehr kritische Haltung der traditionellen Medizin gegenüber der Phytotherapie wider.

\section{Serenoa repens}

2009 erschien ein Review über die Wirksamkeit von Serenoa repens (Sägepalme) bei benigner Prostatahyperplasie (BPH) [1] und hat der Arzneipflanze die Wirksamkeit abgesprochen. Die Übersichtsarbeit überprüfte Studien, die Präparate aus $S$. repens ohne nähere Angaben und Qualitätsstandard verwendeten, und sogar einige Studien, bei denen das Prüfpräparat neben $S$. repens noch weitere Inhaltsstoffe enthielt. Weiter wurden auch Studien eingeschlossen, bei denen $S$. repens mit Tamsulosin und Placebo verglichen wurden. Die Autoren des Cochrane Review schlossen aus den Resultaten, dass $S$. repens einerseits gleich wirkt wie Tamsulosin, andererseits, dass zwischen S. repens und Placebo kein Wirkungsunterschied bestehe. Daraus folgerten sie, $S$. repens sei unwirksam.
Wegen dieser einseitigen Schlussfolgerung und grosser methodischer Fehler wurde die Studie von Fachleuten der Phytotherapie stark angegriffen und die Resultate wurden in Zweifel gezogen.

\section{Weissdorn}

Auf der anderen Seite hat ein anderer, ebenfalls 2009 erschienener Review dem Weissdorn (Crataegus) bei chronischen Herzbeschwerden Wirksamkeit beschieden [2]. Die Autoren der Übersicht werteten die Daten von 14 Studien aus, in denen Patienten mit NYHA(New York Heart Association)-Klassifikation I-III behandelt wurden. Sie folgerten aus den ermittelten Daten: «Diese Resultate legen die Vermutung nahe, dass bei der Verwendung von Weissdornextrakt als Adjuvans zur Behandlung von chronischen Herzbeschwerden ein signifikanter Nutzen zur Verbesserung der Symptome und physiologischer Werte vorliegt.»

Allerdings wirken Weissdornpräparate gemäss dieser Schlussfolgerung nur als Adjuvans, obwohl zahlreiche Studien die Wirksamkeit von Weissdornpräparaten auch als Monotherapie dokumentiert haben.

\section{Artischockenblätter-Extrakt}

Kürzlich ist ein weiterer Review erschienen, der die Wirksamkeit von Artischockenblätter-Extrakt bei Hypercholesterinämie untersuchte [3].
(๑) 2014 S. Karger GmbH, Freiburg 
Dazu werteten die Autoren der Übersicht drei klinische Studien aus und folgerten: «Es gibt einen Hinweis, dass Artischockenblätter-Extrakt ein Potenzial zur Verminderung des Cholesterinspiegels besitzt. Aber die bestehende Evidenz ist noch nicht überzeugend, um Artischockenblätter-Extrakt als eine Behandlungsoption gegen Hypercholesterinämie zu empfehlen.»

Diese Schlussfolgerung der Autoren mag als eher negativ erscheinen. Wenn man sich aber darüber bewusst ist, wie viel erforderlich ist, bis ein Cochrane Review einer Arzneipflanze überhaupt eine gewisse Wirksamkeit zugesteht, dann kann man dieses Urteil auch positiv bewerten! Ausserdem ist zu beachten, dass Phytotherapie-Fachleute die Artischocke nicht in erster Linie zur Cholesterinsenkung empfehlen, sondern gegen funktionelle Dyspepsie.

\section{Cimicifuga}

2012 erschien eine von der Cochrane Collaboration in Auftrag gegebene Studie [4] über die Wirksamkeit der Traubensilberkerze (Cimicifuga racemosa) bei menopausalen Beschwerden und stellte sich mit ihrem negativen Resultat gegen viele publizierte Studien mit einem positiven Resultat. Immerhin gestehen die Cochrane-Autoren ein, dass weitere Studien nötig seien, um die Frage der Wirksamkeit genauer abzuklären.

\section{Ginkgo biloba}

Die Wirksamkeit von Ginkgo bilo$b a$ bei kognitiven Störungen und Demenz wurde in einem 2009 erschienenen Review [5] untersucht. Auch hier gelangten die Autoren zu einem negativen Resultat: «Die Evidenz eines berechenbaren und klinisch signifikanten Nutzens von Ginkgo biloba bei Demenz oder kognitiven Störungen ist widersprüchlich und unsicher.»

Eine genaue Auswertung dieses Reviews wäre nötig, um herauszufinden, warum auch diese Übersicht zu einem negativen Resultat kam, gibt es doch Dutzende von anerkannten Studien, die die Wirksamkeit von G. biloba, insbesondere der Spezialextrakte EGb 761 und Li 1370, hinreichend dokumentieren.

\section{Johanniskraut}

Als einziger Arzneipflanze bescheinigt die Cochrane Collaboration dem Johanniskraut (Hypericum perforatum) uneingeschränkt eine klinische Wirksamkeit, die sogar mit synthetischen Antidepressiva vergleichbar ist. Die entsprechende Übersicht wurde 2008 publiziert [6] und erwähnt auch die gegenüber synthetischen Antidepressiva geringere Anzahl von Nebenwirkungen von Hypericum-Präparaten. Bemerkenswert ist, dass der Erstautor dieses Cochrane Review Klaus Linde ist, ein Phytotherapie-Fachmann mit internationalem Ansehen.

\section{Zusammenfassung}

In diesem Beitrag wurden die Resultate von sechs Cochrane Reviews vorgestellt, die die Wirksamkeit anerkannter Arzneipflanzen untersuchten. Ausser im Falle von Artischockenblätter-Extrakt untersuchten die Autoren der Reviews die Wirksamkeit der überprüften Arzneipflanzen für die Indikationen, die auch von anerkannten Phytotherapie-Fachleuten als die hauptsächlichsten Indikationen bezeichnet werden.

Im Falle von S. repens bei BPH, von C. racemosa bei menopausalen Beschwerden und von G. biloba bei kognitiven Störungen und Demenz sprechen die Autoren dieser Reviews den Arzneipflanzen jede Wirksamkeit ab und befinden sich damit bei allen drei Arzneipflanzen im Widerspruch zu zahlreichen Positivstudien, die von international anerkannten Fachleuten der Phytotherapie durchgeführt wurden. Genauere Abklärungen über die Methodik dieser Cochrane Reviews wären nötig, um die Gründe für diese negativen Resultate zu erfahren.
Im Falle von Weissdorn bei chronischen Herzbeschwerden und von Johanniskraut bei Major-Depressionen erkennt der entsprechende Cochrane Review die Wirksamkeit der entsprechenden Arzneipflanze an. Zudem wird der Artischocke zur Behandlung der Cholesterinämie, die für diese Arzneipflanze nicht die Hauptindikation ist, eine gewisse Wirksamkeit zugesprochen.

\section{Kommentar}

Diese sechs Reviews der Cochrane Collaboration spiegeln exemplarisch die Haltung der Mehrheit der Fachleute der traditionellen Medizin gegenüber der Phytotherapie wider. Viele von ihnen begegnen ihr mit grosser Skepsis und mangelnden Fachkenntnissen, was sich in Studien ausdrückt, die methodische Fehler aufweisen. So zum Beispiel, wenn in einer MetaAnalyse Studien berücksichtigt werden, die keine genauen Angaben über die verwendete Arzneipflanze machen oder sogar Präparate einschliessen, die neben der Arzneipflanze noch weitere Inhaltsstoffe aufweisen.

Ein sehr interessanter Ansatz wäre auch, eine Übersicht über alle Cochrane Reviews zu erstellen, die die Wirksamkeit pflanzlicher Arzneimittel untersuchten.

\section{Literatur}

Tacklind J, MacDonald R, Rutks I, Wilts TJ: Serenoa repens for benign prostatic hyperplasia. Cochrane Database Syst Rev 2009;2: CD001423.

- 2 Guo R, Pittler MH, Ernst E: Hawthorn extract for treating chronic heart failure. Cochrane Database Syst Rev 2008;1:CD005312.

- 3 Wider B, Pittler MH, Thompson-Coon J, Ernst E: Artichoke leaf extract for treating hypercholesterolaemia. Cochrane Database Syst Rev 2009;4:CD003335.

4 Leach MJ, Moore V: Black cohosh (Cimicifuga spp.) for menopausal symptoms. Cochrane Database Syst Rev 2012;9:CD007244.

5 Birks J, Grimley Evans J: Ginkgo biloba for cognitive impairment and dementia. Cochrane Database Syst Rev 2009;1:CD003120.

6 Linde K, Berner MW, Kriston L: St. John's wort for major depression. Cochrane Database Syst Rev 2008;4:CD000448. 